\title{
Radical Surgical Treatment of A Large Seminal Vesicle Cyst Causing Acute Urinary Retention in A Patient with Zinner Syndrome: A Case Report and Review of Literature
}

\author{
(1) Dejan Djordjevic ${ }^{1}$, (1) Svetomir Dragicevic2, (1) Marko Vukovic ${ }^{1}$ \\ ${ }^{1}$ Clinic of Urology, Euromedic General Hospital, Belgrade, Serbia \\ ${ }^{2}$ Clinic of Urology, Clinical Centre of Montenegro, Podgorica, Montenegro
}

\begin{abstract}
Herein, we report a case of a large and complex seminal vesicle cyst causing urinary retention in a young patient with left renal agenesis, psychotic disorder, and deep venous thrombosis. Abdominal computed tomography showed left renal agenesis with associated incipient hydronephrosis of the right renal unit. Furthermore, the whole pelvis was occupied by a large, heterodense cyst $(116 \times 113 \times 107 \mathrm{~mm})$, with several adherent and small cystic lesions $(39 \times 70 \mathrm{~mm})$, compressing the posterior bladder wall. Open surgical approach was performed, including bilateral vesiculectomy with resection of the vas deferentia, since these structures were closely adhering to the cystic wall, and there was no clear surgical plane between them. A brief review of the literature on the main surgical options and postoperative outcomes was also undertaken.
\end{abstract}

Keywords: Zinner syndrome, seminal vesicle cyst, surgical treatment

\section{Introduction}

Zinner syndrome is a congenital anomaly comprising renal agenesis, seminal vesicle cysts, and ejaculatory duct obstruction $(1,2)$. In symptomatic cases, patients may complain of lower urinary tract symptoms, including epididymitis and postejaculation pain. These symptoms are reported in approximately $30 \%$ of patients and usually related to the mass effect of the cyst during sexual activities (1-4). Conservative treatment is usually feasible for most cases; however, minimally invasive procedures may be considered as well $(5,6)$. In challenging cases, surgical resection of the cyst by laparoscopic or open surgery may be necessary. Open surgery has been the preferred approach for many decades since good postoperative results have been reported for the preservation of genital structures. Moreover, a lower abdominal midline incision provides excellent tissue exposure, and it remains the most suitable approach in patients with large seminal vesicle cysts (4). Despite its advantages, open excision may be associated with injuries to pelvic organs such as the rectum, bladder, and pelvic ureter or formation of urinoma (7).
The current literature presented only a few cases of complex cystic lesions with invasion of surrounding structures, impeding fertility after surgical treatment. Herein, we present the radical surgical treatment of a unique case of complex, large, and infiltrative seminal vesicle cyst in a patient with Zinner syndrome, describing an unusual approach in a young, sexually active patient.

\section{Case Reports}

A 34-year-old Caucasian man, without children, was referred to our clinic in October 2020 for urodynamic testing following an episode of acute urinary retention. Before referral to our institution, the report of his previous urologist was unremarkable, except for noting a distended bladder.

The patient complained of dysuria and perineal discomfort for 2 weeks, without fever or any other symptoms. His left renal agenesis was diagnosed many years ago by computed tomography (CT). He also had experienced a few episodes of acute paranoid reaction, although he was presently in remission. The

Correspondence: Marko Vukovic MD, Clinic of Urology, Clinical Centre of Montenegro, Podgorica, Montenegro

Phone: +382 69498879 E-mail: marko.vukovic09@gmail.com ORCID-ID: orcid.org/0000-0003-0265-4721

Received: 31.01 .2021

Accepted: 19.07.2021

Cite this article as: Djordjevic D, Dragicevic S, Vukovic M. Radical Surgical Treatment of A Large Seminal Vesicle Cyst Causing Acute Urinary Retention in A Patient with Zinner Syndrome: A Case Report and Review of Literature. J Urol Surg 2021;8(4):291-293.

${ }^{\circ}$ Copyright 2021 by the Association of Urological Surgery / Journal of Urological Surgery published by Galenos Publishing House. 
physical examination was unremarkable, and the vas deferens was bilaterally palpable. During a digital rectal examination, an irregular, partially fluctuant and partially solid mass was palpated in the prostatic fossa, measuring approximately $10 \mathrm{~cm}$ in diameter; the mass was painless and had no clear borders to the surrounding structures.

Laboratory analyses of the mass showed high levels of D-dimer $(>1000 \mu \mathrm{g} / \mathrm{L})$ and creatinine $(124 \mu \mathrm{mol} / \mathrm{L})$. Urinalysis and other blood tests were unremarkable. On further investigation, Doppler ultrasonography of the lower extremity revealed old thrombotic masses within the common and superficial right femoral veins, with signs of recanalization. Abdominal CT confirmed left renal agenesis associated with incipient hydronephrosis of the right renal unit, with multiple ureteral "kinking" (Figure 1a). Furthermore, the whole pelvis was occupied by a large, heterodense cyst $(116 \times 113 \times 107 \mathrm{~mm})$, with several adherent and small cystic lesions $(39 \times 70 \mathrm{~mm})$, which resembled a morphologically degenerated left seminal vesicle (Figure 1b). The cyst had compressed the posterior bladder wall, and its volume was reduced significantly (Figure 1c). The right seminal vesicle could not be clearly detected (confined within the cystic lesion), and the prostate appeared normal, as it was clearly defined and homogenous.

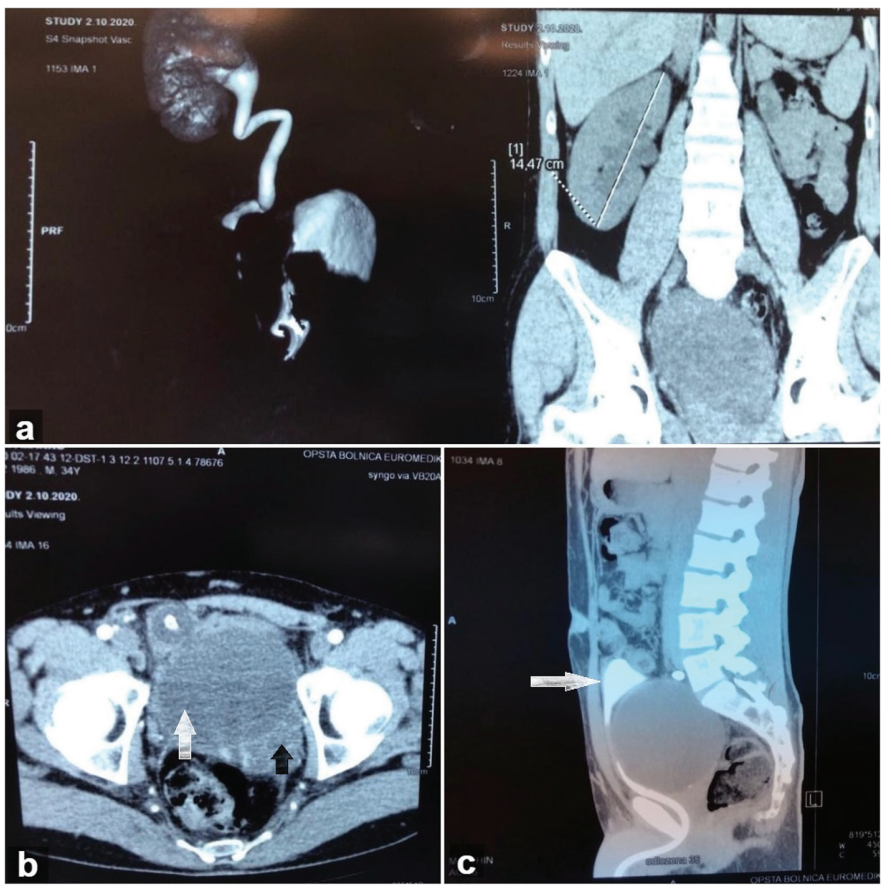

Figure 1. (a) Abdominal computed tomography with image reconstruction shows left renal agenesis and multiple right ureteral "kinkings" with incipient ureterohydronephrosis; (b) a large, heterodense (white arrow) and multifocal seminal vesicle cyst (black arrow), with anterior displacement of the bladder; (c) delayed phase CT urography reveals mass effect of seminal vesicle cysts with significant reduction of bladder capacity (white arrow). Contrast is seen within the bladder and urethral catheter

\section{Surgical Treatment}

An open surgical approach was initiated, as conservative treatment was not an option. The patient was positioned supine, and a lower midline incision was made to allow for an extraperitoneal approach. After separating the rectus muscle fibers, a large cystic mass was identified, bulging from the wound deep within the pelvis (Figure 2a). The cyst was multilocular in its distal part, with no clear plane of dissection to the left seminal vesicle. Moreover, the vas deferens was adherent to the cystic wall bilaterally, without a clear surgical plane between these structures (Figure 2b). Although the cyst was completely separated from the prostate, further dissection from the ipsilateral seminal vesicle and vas deferens was unsuccessful because of the close proximity of these structures to the cystic membrane. Therefore, bilateral vesiculectomy with resection of the vas deferentia was inevitable (Figure 2c). In the early postoperative period, the patient developed repeated urinary retention, which was treated with Foley catheterization. Cystoscopy revealed increased bladder capacity $(>1500 \mathrm{~mL})$, without voiding desire, even after instillation of $1500 \mathrm{~mL}$ of sterile saline. In the following month, urodynamic testing revealed normal detrusor function, with no involuntary voiding. The final pathology report confirmed the diagnosis of a complex cyst with nodular steatonecrosis, but no signs of malignancy.

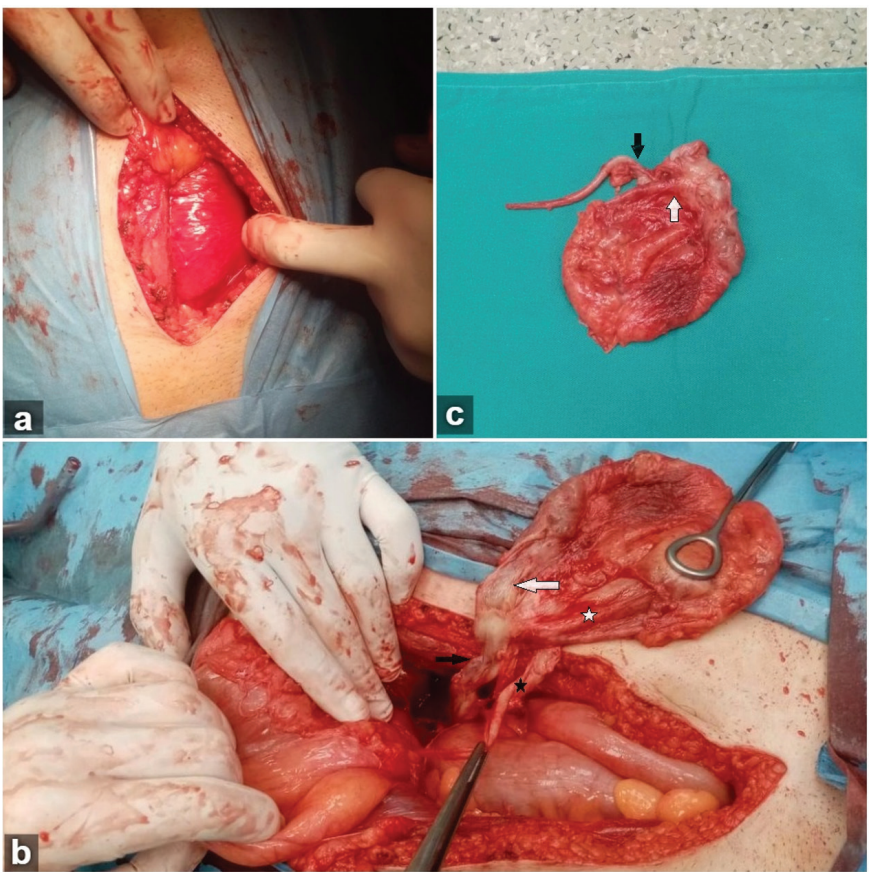

Figure 2. (a) A large cystic mass emerging from the deep pelvis; (b) a large cystic mass emerging from the left seminal vesicle (white arrow) with dilated vas deferens (black arrow); right seminal vesicle (white star) and distal vas (black star) adherent to the cystic wall; (c) resected specimen: left seminal vesicle and vas deferens (white arrow), together with right the vas deferens (black arrow). The right seminal vesicle is not seen on this image 


\section{Discussion}

Herein, we present an unusual congenital anomaly, which in most instances, is difficult to detect. Only a few cases have been reported in the literature, and most cases presented during the sexually active period of life, usually in association with infertility $(1,8)$. According to Tan et al. $(2)$, seminal vesicle cysts can be categorized into four groups, ranging from simple cysts treated by a transrectal or transperineal approach to potentially malignant lesions, which are best treated by an open surgical resection. Our case report presents a complex cyst, which was larger than $10 \mathrm{~cm}$, was multilocular and heterodense on $\mathrm{CT}$, and required surgical treatment. In addition, the indication for surgery was compressive effect of the mass on the bladder (2).

Observation management may be implemented in asymptomatic or slightly symptomatic cases (8). Therefore, even symptomatic cases may benefit from conservative management with intense follow-up if the ejaculatory duct is unobstructed and the ipsilateral testis remains normal (9). Invasive treatment should be restricted to challenging cases or patients whose treatment fails using conservative measures (6). A conservative transrectal aspiration, although easy to perform, is associated with a high risk of recurrence and infection and should not be repeated if ineffective (1). Laparoscopic or robotic approaches are preferred, since they provide the best results in terms of blood loss and hospital stay $(10,11)$.

However, in this case, we performed bilateral open vesiculectomy and resected the vas deferentia without prior clinical confirmation of infertility. However, this was the only treatment option available, as a large, multilocular cystic lesion had involved both seminal vesicles, and a clear dissection plane was not possible. Moreover, this patient had a history of psychiatric disorders, which presented as an additional risk factor for infertility in the preoperative setting. Thus, we determined that partial vesiculectomy or other conservative approaches should not be considered in patients with large infiltrative cysts and unproven fertility, especially if any concern exists regarding the oncological safety and completeness of cystic resection. Finally, assisted reproduction may be offered to the patient with preserved testicular function.

This case points out the necessity of radical surgery in young male patients with Zinner syndrome accompanied by a large, complex, infiltrative seminal vesicle cyst despite its detrimental effect on potential fertility.

\section{Ethics}

Informed Consent: Patient gave her consent prior to the creation of this case report.
Peer-review: Externally peer-reviewed.

\section{Authorship Contributions}

Surgical and Medical Practices: M.V., D.D., S.D., Concept: M.V., D.D., S.D., Design: M.V., D.D., S.D., Data Collection or Processing: M.V., D.D., S.D., Analysis or Interpretation: M.V., D.D., S.D., Literature Search: M.V., D.D., S.D., Writing: M.V., D.D., S.D.

Conflict of Interest: No conflict of interest was declared by the authors.

Financial Disclosure: The authors declared that this study received no financial support.

\section{References}

1. Demaeyer L, Holz S, Pamart D, Taylor S, Naudin M. Robotic management of painful Zinner syndrome, case report and review of literature. Int J Surg Case Rep 2020;73:61-64.

2. Tan Z, Li B, Zhang L, Han P, Huang H, Taylor A, Li X. Classifying seminal vesicle cysts in the diagnosis and treatment of Zinner syndrome: $A$ report of six cases and review of available literature. Andrologia 2020;52:e13397.

3. Fiaschetti V, Greco L, Giuricin V, De Vivo D, Di Caprera E, Di Trapano R, Castellani $F$, Floris $R$. Zinner syndrome diagnosed by magnetic resonance imaging and computed tomography: role of imaging to identify and evaluate the uncommon variation in development of the male genital tract. Radiol Case Rep 2016;12:54-58.

4. van den Ouden D, Blom JH, Bangma C, de Spiegeleer AH. Diagnosis and management of seminal vesicle cysts associated with ipsilateral renal agenesis: a pooled analysis of 52 cases. Eur Urol 1998;33:433-440.

5. Naval-Baudin P, Carreño García E, Sanchez Marquez A, Valcárcel José J, Romero NM. Multicystic seminal vesicle with ipsilateral renal agenesis: two cases of Zinner syndrome. Scand J Urol 2017;51:81-84.

6. Pereira $B J$, Sousa $L$, Azinhais $P$, Conceição $P$, Borges $R$, Leão $R$, Brandão $A$, Temido $P$, Retroz E, Sobral F. Zinner's syndrome: an up-to-date review of the literature based on a clinical case. Andrologia 2009;41:322-330.

7. Politano VA, Lankford RW, Susaeta R. A transvesical approach to total seminal vesiculectomy: a case report. J Urol 1975;113:385-388.

8. Hevia Palacios M, Álvarez-Maestro M, Gómez Rivas J, Aguilera Bazan A, Martinez-Piñeiro L. Zinner Syndrome with Ectopic Ureter Emptying into Seminal Vesicle. Case Rep Urol 2021;2021:8834127.

9. Manousakas T, Kyriakou G, Serafetinides E, Giannopoulou M, Kyroudi A, Giannopoulos A. Partial vesiculectomy in an infertile man with seminal vesicle cyst, ipsilateral renal agenesis, and cryptorchidism. Urology 2002;59:602.

10. Selli C, Cavalleri S, De Maria M, lafrate M, Giannarini G. Robot-assisted removal of a large seminal vesicle cyst with ipsilateral renal agenesis associated with an ectopic ureter and a Müllerian cyst of the vas deferens. Urology 2008;71:1226.e5-1227.

11. Kord E, Zisman A, Darawsha AE, Dally N, Noh PH, Neheman A. Minimally Invasive Approach for Treatment of Seminal Vesicle Cyst Associated with Ipsilateral Renal Agenesis. Urol Int 2017;99:338-342. 